\title{
DNA damage triggers nucleotide excision repair-dependent monoubiquitylation of histone H2A
}

\author{
Steven Bergink, ${ }^{1}$ Florian A. Salomons, ${ }^{3}$ Deborah Hoogstraten,, ${ }^{1,3}$ Tom A.M. Groothuis, ${ }^{5}$ \\ Harm de Waard, ${ }^{1}$ Junxin $\mathrm{Wu},{ }^{3} \mathrm{Li}$ Yuan, ${ }^{3}$ Elisabetta Citterio ${ }^{4}{ }^{2}$ Adriaan B. Houtsmuller, ${ }^{2}$ \\ Jacques Neefjes, ${ }^{5}$ Jan H.J. Hoeijmakers, ${ }^{1}$ Wim Vermeulen, ${ }^{1,7}$ and Nico P. Dantuma ${ }^{3,6}$ \\ ${ }^{1}$ MGC Department of Cell Biology and Genetics, Center for Biomedical Genetics, and ${ }^{2}$ Department of Pathology, Erasmus \\ Medical Center, Rotterdam 3015GE, The Netherlands; ${ }^{3}$ Department of Cell and Molecular Biology, The Medical Nobel \\ Institute, Karolinska Institutet, Stockholm S-17177, Sweden; ${ }^{4}$ IFOM, Istituto FIRC di Oncologia Molecolare, Milan I-20139, \\ Italy; ${ }^{5}$ Division of Tumor Biology, The Netherlands Cancer Institute, Amsterdam 1066CX, The Netherlands
}

\begin{abstract}
Chromatin changes within the context of DNA repair remain largely obscure. Here we show that DNA damage induces monoubiquitylation of histone H2A in the vicinity of DNA lesions. Ultraviolet (UV)-induced monoubiquitylation of $\mathrm{H} 2 \mathrm{~A}$ is dependent on functional nucleotide excision repair and occurs after incision of the damaged strand. The ubiquitin ligase Ring2 is required for the DNA damage-induced H2A ubiquitylation. UV-induced ubiquitylation of H2A is dependent on the DNA damage signaling kinase ATR (ATM- and Rad3-related) but not the related kinase ATM (ataxia telangiectasia-mutated). Although the response coincides with phosphorylation of variant histone $\mathrm{H} 2 \mathrm{AX}, \mathrm{H} 2 \mathrm{AX}$ was not required for $\mathrm{H} 2 \mathrm{~A}$ ubiquitylation. Together our data show that monoubiquitylation of $\mathrm{H} 2 \mathrm{~A}$ forms part of the cellular response to $\mathrm{UV}$ damage and suggest a role of this modification in DNA repair-induced chromatin remodeling.
\end{abstract}

[Keywords: DNA repair; ubiquitin-proteasome system; histone; chromatin; ATR; H2AX; DNA damage response]

Supplemental material is available at http://www.genesdev.org.

Received November 22, 2005; revised version accepted March 13, 2006.

Proper cellular functioning depends on the maintenance of the genetic and epigenetic information. The integrity of these constituents is, however, constantly jeopardized by genotoxic agents both from endogenous sources such as reactive oxygen species and from exogenous sources including radiation. A complex network of DNA damage response (DDR) mechanisms collectively protects genome integrity, including several DNA repair pathways and damage-induced cell cycle checkpoints (Hoeijmakers 2001; Peterson and Cote 2004). The nucleotide excision repair (NER) machinery is involved in the removal of a wide variety of helix-distorting DNA lesions, such as cyclobutane pyrimidine dimers (CPD) and 6-4 photoproducts, which are introduced by exposure to ultraviolet (UV) light (de Laat et al. 1999). More than 30 proteins are involved in NER. Xeroderma pigmentosum (XP) patients have a NER deficiency due to mutations in DNA repair proteins and typically suffer from photosensitivity

Corresponding authors.

${ }^{6} \mathrm{E}-\mathrm{MAIL}$ nico.dantuma@ki.se; FAX 46-8-313529.

${ }^{7}$ E-MAIL w.vermeulen@erasmusmc.nl; FAX 31-10-4089468.

Article and publication are at http://www.genesdev.org/cgi/doi/10.1101/ gad.373706. and predisposition to skin cancer. Seven NER genes responsible for XP have been identified, designated XPA to $X P G$ (de Laat et al. 1999). Depending on the manner in which the lesions are recognized, NER can be subdivided in global genome NER (GG-NER) and transcriptioncoupled NER (TC-NER). Whereas the latter is confined to transcriptional active regions where a lesion-stalled RNA polymerase sets off the NER response (Citterio et al. 2000), GG-NER is initiated by genome-wide recognition of lesions by the consorted action of the UV-damaged DNA-binding (DDB) complex and the XPC/ hHR23B/Cen2 complex (Sugasawa et al. 1998; Araki et al. 2001).

In eukaryotic cells, the DNA is tightly packed in chromatin, consisting of highly organized nucleosomes. The tails of histones are subject to a wide range of post-translational modifications such as acetylation, methylation, phosphorylation, and ubiquitylation and play a central role in the regulation of chromatin activity (Jenuwein and Allis 2001). Transcription, replication, recombination, and DNA repair are intimately connected with histone modifications, which regulate accessibility of the DNA as well as recruitment of important factors. In mammals, the C-terminal tail of histone $\mathrm{H} 2 \mathrm{~A}$ is a promi- 
nent target for ubiquitin resulting in as much as $5 \%-$ $15 \%$ of $\mathrm{H} 2 \mathrm{~A}$ being monoubiquitylated (Zhang 2003). The ubiquitin ligase Ring2 was recently shown to be the dominant ubiquitin ligase of histone $\mathrm{H} 2 \mathrm{~A}$ (de Napoles et al. 2004; Wang et al. 2004). Ubiquitylated H2A (uH2A) is linked to condensed DNA and associated with repression of gene expression (Levinger and Varshavsky 1982), X-chromosome inactivation (de Napoles et al. 2004), and polycomb gene silencing (Wang et al. 2004).

A number of histone modifications are implicated in DNA repair including phosphorylation, acetylation, and methylation (Vidanes et al. 2005). It has been suggested that chromatin is remodeled during DNA repair and transits between different stages that subsequently allow factors to access and repair the lesion after which the original state is restored (Smerdon 1991). Moreover, histone modifications play a pivotal role in DNA damageinduced signaling responsible for activating checkpoints that will pause cell cycle progression until the genome integrity has been restored (Green and Almouzni 2002; Vidanes et al. 2005). A well characterized DNA damageinduced histone modification is phosphorylation of the variant histone H2AX (Thiriet and Hayes 2005). H2AX phosphorylation is dependent on the DDR kinases ataxia telangiectasia-mutated (ATM) and ATM- and Rad3-related (ATR) that are activated after double-strand breaks, replication blocks, and UV light, respectively (Shiloh 2003). Although phosphorylated H2AX ( $\gamma \mathrm{H} 2 \mathrm{AX})$ has originally been identified as a marker for double-strand breaks (Rogakou et al. 1998), it is also induced in a NERdependent manner in UV-exposed cells (O'Driscoll et al. 2003). In sharp contrast to the plethora of histone modifications involved in regulation of chromatin activity, H2AX phosphorylation is the only presently known NER-dependent histone modification in higher eukaryotes (O'Driscoll et al. 2003).

It has become clear that ubiquitylation plays a pivotal role in DNA repair (Hoege et al. 2002; Kannouche et al. 2004; Sugasawa et al. 2005). To gain more insight in this exciting link, we set up a system for following ubiquitylation in living cells in response to genotoxic stress. Here, we show that UV exposure of human cells causes a significant NER-dependent monoubiquitylation of histone H2A. This novel DNA damage-induced histone ubiquitylation event reveals an additional link between the ubiquitin system and DNA repair.

\section{Results}

\section{DNA damage induces accumulation of ubiquitin}

In order to study ubiquitylation in living cells in response to genotoxic stress, ubiquitin was tagged at its $\mathrm{N}$ terminus with the green fluorescent protein (GFP). The resulting GFP-ubiquitin (Ub) fusion is functionally competent and properly conjugated to target proteins (Qian et al. 2002; Dantuma et al. 2006). This construct was stably introduced in the human melanoma cell line Mel JuSo. We noticed that upon local irradiation of the nuclei of GFP-Ub cells with an intense 405-nm laser, the initial bleaching of the GFP fluorophore in the exposed region was rapidly followed by local accumulation of fluorescent GFP-Ub indicative for recruitment of GFP-Ub to the laser exposed region (Fig. 1A). Accumulation of GFP-Ub after laser exposure coincided with sequestration of PCNA (proliferation cell nuclear antigen), suggesting a link with DNA repair (see below). To study a more defined type of DNA damage, we inflicted local UV-C damage in subnuclear areas (Katsumi et al. 2001). Locally UV-exposed cells revealed accumulation of GFP-Ub at the damaged areas, which were identified by the presence of CPD lesions (Fig. 1B, top) and colocalized with the early NER damage recognition factor XPC (Fig. 1B, bottom). Importantly, a similar staining pattern was observed for endogenous ubiquitin in locally UV-irradiated wild-type fibroblasts (Fig. 1C), indicating that this behavior is not caused by a nonspecific property of GFP-Ub.

To examine whether ubiquitin is recruited to the site of DNA damage through specific conjugation, we used the mutant ubiquitin $\mathrm{Ub}^{\mathrm{KO}, \mathrm{G} 76 \mathrm{~V}}$ fused to GFP. In this conjugation-incompetent mutant $\mathrm{Ub}$, all seven lysine residues, which are potentially involved in the formation of polyubiquitin chains, as well as the C-terminal glycine residue that is crucial for ubiquitin conjugation have been replaced by arginines and valine, respectively (Qian et al. 2002; Dantuma et al. 2006). The mutant GFP-Ub ${ }^{\mathrm{KO}, G 76 V}$ failed to accumulate at the sites of lesions induced by local UV irradiation (Fig. 1D) or laser exposure (data not shown). Thus, DNA damage induced by UV or laser light exposure initiates rapid local ubiquitin conjugation.

\section{DNA damage causes monoubiquitylation without proteasome recruitment}

To investigate which type of ubiquitylation was responsible for the observed UV-induced response, we used the mutant GFP-Ub ${ }^{\mathrm{KO}}$. This mutant acts as a chain terminator since it lacks lysine residues to further extend polyubiquitin chains. Therefore, this ubiquitin mutant can efficiently participate in monoubiquitylation events while polyubiquitylation is hindered. We found that both GFP-Ub and GFP-Ub ${ }^{\mathrm{KO}}$ readily accumulated in laser-exposed regions (Fig. 2A). Time lapse imaging showed similar kinetics for the accumulation of each construct, suggesting that the laser light-induced ubiquitin accumulation represents predominantly monoubiquitylated species (Fig. 2B).

Ubiquitin and the proteasome are intimately linked in many processes including DNA repair (Glickman and Ciechanover 2002; Wang et al. 2005). However, the proteasome interacts primarily with polyubiquitin chains (Thrower et al. 2000). Consistent with the idea that DNA damage triggered mainly monoubiquitylation, we found that $20 \mathrm{~S}$ proteasomes carrying the GFP-tagged $\alpha 3$ subunit did not accumulate at local DNA damage (Fig. 2C) nor did we observe a colocalization of endogenous proteasomes in immunostainings (data not shown). Moreover, the mobility of the GFP-tagged proteasomes was not significantly altered in the nuclear compart- 
A

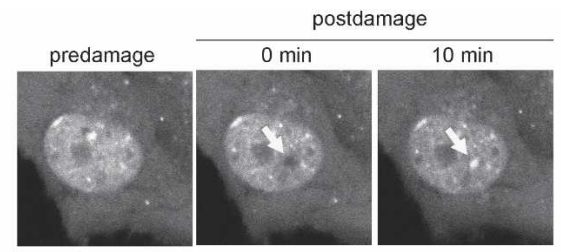

B

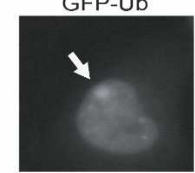

GFP-Ub

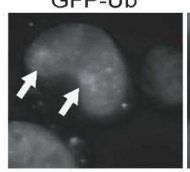

$\alpha-U b$
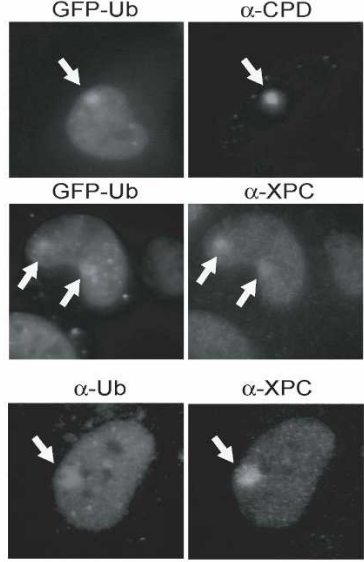

GFP-Ub, $\alpha-C P D$
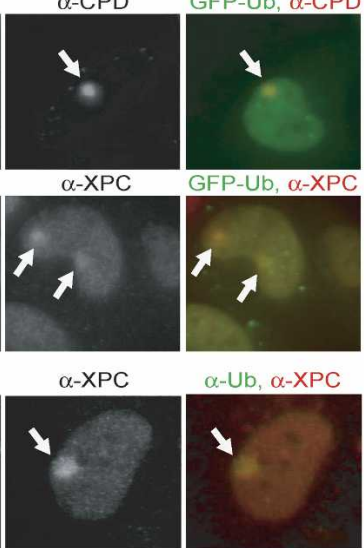

D

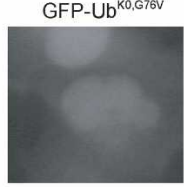

$\alpha-\mathrm{CPD}$

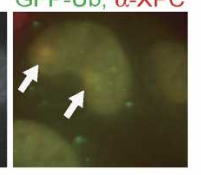

$\alpha-U b, \alpha-X P C$

GFP-Ub ${ }^{\mathrm{K} 0.676 \mathrm{~V}}$

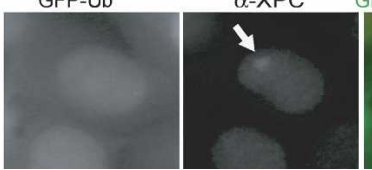

4
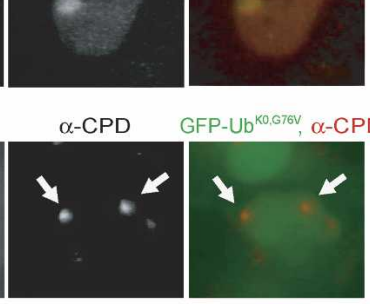

GFP-Ub ${ }^{K 0 . G 76 V}, \alpha-C P D$

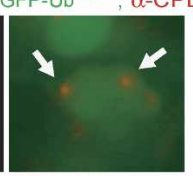

GFP-Ub ${ }^{K 0,67 \% \%} \alpha-X P C$
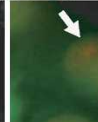

4

$\sin =3$

Figure 1. DNA damage induces accumulation of ubiquitin. $(A)$ Mel JuSo cells stably expressing GFP-Ub were locally irradiated in the nucleus with a high-intensity 405-nm laser beam. Accumulation of GFP-Ub appeared at the local laser-irradiated spot, indicated by the arrow. (B) Mel JuSo cells stably expressing GFPUb were locally irradiated with UV-C light. A clear accumulation of GFP-Ub is visible at the local UV-irradiated areas. The arrows indicate the local UV DNA-damaged spot visualized with either the presence of DNA damage by CPD counterstaining (top middle panel) or the presence of the NER protein XPC (bottom middle panel). Colocalization of GFP-Ub with the DNA damage (top right panel) and XPC protein (bottom right panel) is shown. (C) An antibody recognizing conjugated endogenous ubiquitin revealed accumulation of ubiquitin at local irradiated spots, marked by the presence of XPC protein, in primary human fibroblasts (C5RO). Staining with the ubiquitinspecific antibody (right panel), the XPC-specific antibody (middle panel), and the merged images (left panel) are shown. (D) Ubiquitin accumulation at local DNA damage is dependent on conjugation. The nonfunctional mutant GFP-Ub ${ }^{\mathrm{K} 0, \mathrm{G} 76 \mathrm{~V}}$ (left panel) did not accumulate in Mel JuSo cells at the DNA damage, as detected by CPD (top middle image) or the presence of XPC (bottom middle image).

ments in response to UV exposure, arguing against substantial recruitment of the $20 \mathrm{~S}$ proteasome to the lesions (data not shown).

In order to analyze cell cycle dependency of the ubiq-

uitylation response, we cotransfected Mel JuSo cells with GFP-tagged proliferation cell nuclear antigen (GFPPCNA) and monomeric red fluorescent protein (mRFP)Ub. GFP-PCNA can be used to distinguish cells in $S$ phase from cells in G1 and G2 phase since it localizes on the replication forks during $S$ phase giving rise to a characteristic punctuate nuclear staining (Leonhardt et al. 2000; Essers et al. 2005). We did not observe any striking differences between non-S-phase and S-phase cells in local mRFP-Ub accumulation (Fig. 2D,E). Notably, also GFP-PCNA, which is involved in various DDR mechanisms, was sequestered in the laser-exposed region, further confirming that the 405-nm laser causes DNA damage. We conclude that the DNA damage-induced monoubiquitylation response occurs throughout the cell cycle.

\section{UV-induced local monoubiquitylation is dependent on functional NER}

UV-induced lesions in placental mammals are predominantly repaired in a cell cycle independent manner by NER (Hoeijmakers 2001). Therefore, we investigated whether functional NER was implicated in this process (Fig. 3A). We compared the response of GFP-Ub in wildtype (NER-proficient) and various XP (NER-deficient) fibroblasts. UV-induced ubiquitylation was completely abrogated in two independent XP-C fibroblast cell lines, suggesting that functional GG-NER is required for this ubiquitin modification (Fig. 3B,C; data not shown). The DNA damage accumulation of ubiquitin was also undetectable in XP-A (Fig. 3D), XP-G (Fig. 3E), and XP-F (data not shown) cell lines. The latter two cell lines are deficient in the NER-specific endonucleases that incise the damaged strand $3^{\prime}$ and $5^{\prime}$ of the lesion, respectively. This suggests that the monoubiquitylation occurs during a late NER step.

$\mathrm{XP}$ variant $(\mathrm{XP}-\mathrm{V})$ patients display a similar pathology as NER deficient patients (Cleaver 2005). However, these patients are not defective in NER but carry instead a mutated DNA polymerase $\eta$, one of the translesion (TLS) DNA polymerases involved in replicative bypass of specific DNA lesions (Masutani et al. 1999). In line with a strict NER dependency of the ubiquitylation event, we found that UV exposure of XP-V primary fibroblasts resulted in a similar colocalization of XPC and endogenous ubiquitin on DNA lesions (Fig. 3F), while endogenous ubiquitin did not accumulate on local damage in primary XP-A cells (data not shown).

\section{Global UV irradiation causes NER-dependent immobilization of nuclear ubiquitin}

The fact that GFP-Ub conjugation on lesions is clearly traceable in living cells argues that a significant fraction of the GFP-Ub pool may be involved in this process. To obtain quantitative information of the UV-induced ubiquitylation, the overall mobility of the total nuclear ubiquitin pool before and after DNA damage was investigated by photobleaching experiments. In nonchallenged 
Bergink et al.

Figure 2. DNA damage causes monoubiquitylation without proteasome recruitment. $(A)$ Cells were transfected with either GFP-Ub or the mutant GFP-Ub ${ }^{\mathrm{k} 0}$, which can be efficiently monoubiquitylated but hinders polyubiquitylation. Both GFP-Ub and GFP-Ub ${ }^{\mathrm{KO}}$ localized at the laser-irradiated areas, which are indicated by the arrows. (B) Quantification of fluorescence recovery in the bleached area circled in $A$. Accumulation of GFP-Ub (closed circles) and GFP-Ub ${ }^{\mathrm{KO}}$ (open circles) did not differ, indicating that the observed GFP-Ub accumulation is primarily caused by monoubiquitylation. (C) Proteasomes tagged with $\alpha 3$ GFP fusion subunit were followed in $\mathrm{Mel}$ JuSo cells after high-intensity laser bleaching as in $A$. The circle denotes the bleached spot. The proteasomes did not accumulate at the high laser-irradiated spots. $(D)$ The mRFP-Ub fusion accumulates on local laser-induced damage in G1 or G2 cells as was observed by the GFP-PCNA staining. (E) Similarly, cells with a typical S-phaselike GFP-PCNA staining were locally damaged using the 405 laser; mRFP-Ub accumulated on these sites.
A

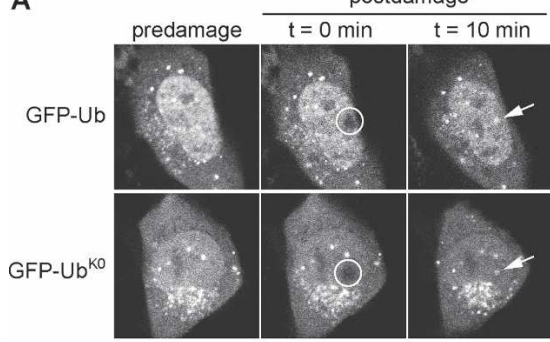

B

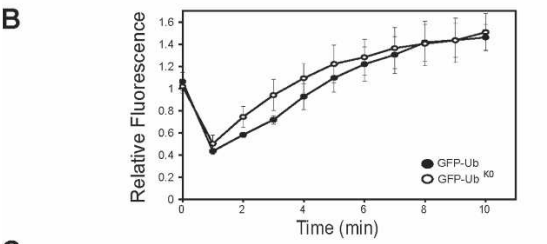

C

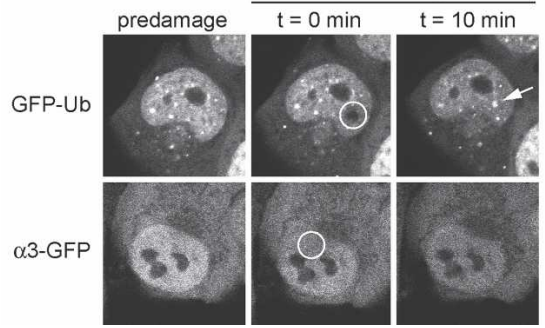

D

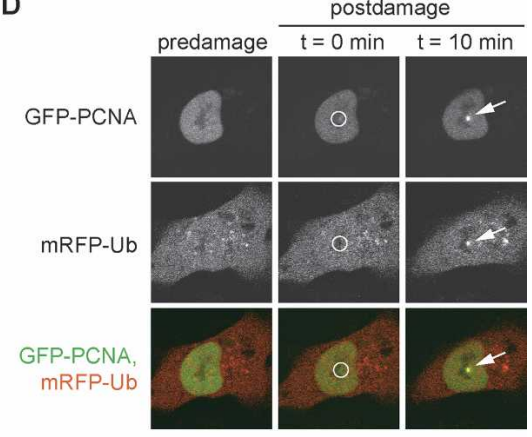

E

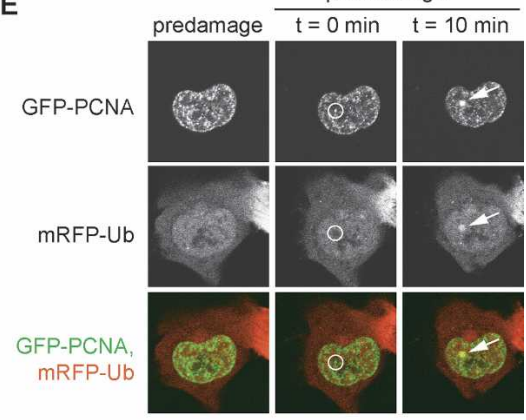

cells a significant immobile fraction of the nuclear GFPUb can be observed by the incomplete recovery of fluorescence in the photobleached area (Fig. 4A). An increase of the immobile pool of GFP-Ub was noted after UV irradiation in a dose-dependent fashion (Fig. 4B). At the maximum dose of $16 \mathrm{~J} / \mathrm{m}^{2}$, we recorded a $43 \%$ increase in the levels of immobile GFP-Ub. Importantly, no such UV-induced increase of immobile GFP-Ub was found in NER-deficient XP-A cells (Fig. 4C) and XP-C cells (data not shown), confirming the functional requirement of NER for the local nuclear accumulation of ubiquitin at the damaged area. Cell lines expressing the conjugationdeficient GFP-Ub ${ }^{\mathrm{KO}, \mathrm{G} 76 \mathrm{~V}}$ mutant did not display the nuclear immobile pool nor did we observe a UV-induced immobilization (Fig. 4D).

\section{Histone $\mathrm{H} 2 \mathrm{~A}$ is the primary target} for UV-induced monoubiquitylation

It has been shown that ubiquitylation plays a role in DNA repair (Hoege et al. 2002; Kannouche et al. 2004; Sugasawa et al. 2005). Interestingly, however, none of the previously described UV-induced ubiquitylation targets are likely candidates for the here described monoubiquitylation. XPC and DDB2 are polyubiquitylated following UV-induced DNA damage (Sugasawa et al. 2005). However, ubiquitylation of XPC or DDB2 does not explain our observation since both these ubiquitylation events occur in wild-type and XPA-deficient cells (Sugasawa et al. 2005). Moreover, we were dealing primarily with a monoubiquitylation event (see Fig. 2B). Another potential target would be PCNA, which is both mono- and polyubiquitylated after damage (Hoege et al. 2002; Kannouche et al. 2004). However, monoubiquitylation of PCNA is linked to TLS and not to NER. Furthermore, PCNA monoubiquitylation is unaffected in XP-A and XP-C fibroblasts (Kannouche et al. 2004). In addition, TLS occurs strictly in S phase, whereas the here described ubiquitylation happens throughout the entire cell cycle (Fig. 2D,E)

We argued that given the dramatic effect on the mobility of nuclear ubiquitin, an abundant immobile nuclear protein would be a likely target. Since histones are immobile, rather abundant and a prominent target for monoubiquitylation (Zhang 2003), we investigated whether UV exposure changed the ubiquitylation status of histones. Probing isolated histones from untreated and UV-irradiated wild-type fibroblasts with a ubiquitin antibody revealed a clear increase in a band of $23 \mathrm{kDa}$ corresponding in size to monoubiquitylated $\mathrm{H} 2 \mathrm{~A}$ (Fig. 5A). The same band reacted with an antibody directed against uH2A (Fig. 5B) and with an H2A-specific antibody (data not shown). Analysis of a tryptic digest from the corresponding band by liquid chromatography tandem mass spectroscopy revealed three peptides originating from ubiquitin and two peptides originating from H2A (Fig. 5C). Immunocytochemical analysis of locally inflicted UV lesions in wild-type fibroblasts with the uH2A-specific antibody confirmed the enrichment of endogenous uH2A at the damaged area (Fig. 5D), while locally damaged areas in XP-A fibroblasts were not marked by this antibody (Fig. 5E).

Normal nondamage-induced monoubiquitylation of histone H2A occurs at Lys 119 (Nickel and Davie 1989). 
A

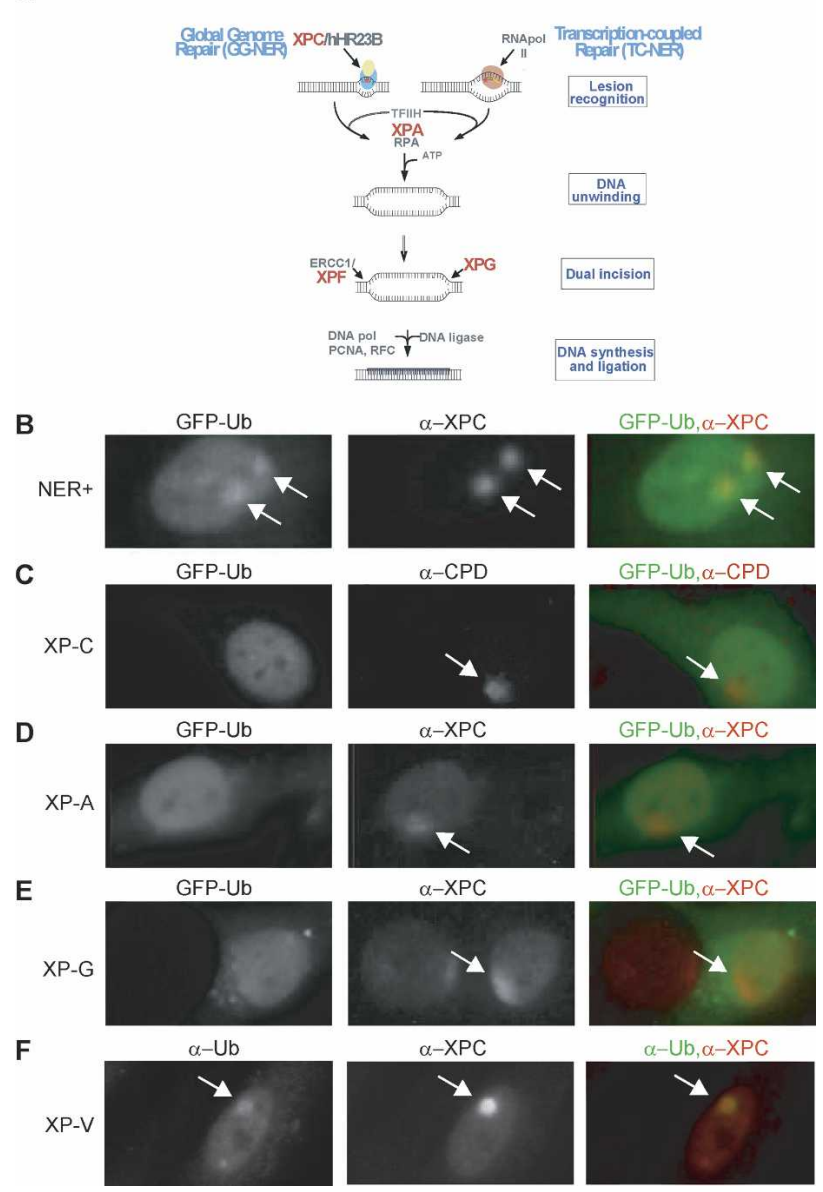

Figure 3. UV-induced local monoubiquitylation is dependent on functional NER. (A) A schematic drawing of the NER pathway is shown. NER factor deficiencies that have been tested in this study are indicated in red. NER lesion recognition is predominantly performed by the XPC complex, after which the TFIIH complex and XPA and RPA are recruited, probably proceeded by XPG. The DNA helix is unwound by the TFIIH complex in an ATP-dependent fashion. The two endonucleases, XPG and XPF-ERCC1, excise the lesion. After incision, the GAP is filled in by DNA polymerase $\delta / \varepsilon$, PCNA, and RFC and is sealed by ligase I (Hoeijmakers 2001). (B) In normal fibroblasts (C5RO-hTERT), GFP-Ub (left panel) accumulated at local damaged spots, indicated by the arrow. (C) In XP-C human fibroblast (XP21RO-Sv), GFP-Ub (left panel) did not accumulate at local damaged spots as detected by CPD (middle panel). (D) GFP-Ub (left panel) did not accumulate in XP-A cells (XP12RO-Sv) at NER active sites as detected by XPC (middle panel). (E) In XP-G (XP3BR-Sv), GFP-Ub (left panel) did not accumulate at local UV-irradiated spots as indicated by the presence of XPC protein (middle panel). (F) In primary NER proficient XP-V cells (XP1RO), deficient in TLS polymerase $\eta$, ubiquitin accumulated at the UV-induced lesions.

To determine whether this lysine residue was also targeted for ubiquitylation upon UV exposure, we expressed Flag-tagged $\mathrm{H} 2 \mathrm{~A}$ ( ${ }^{\text {Flag }} \mathrm{H} 2 \mathrm{~A}$ ) and Flag-tagged H2A in which Lys 119 had been substituted by an arginine residue ( ${ }^{\text {Flag }} \mathrm{H} 2 \mathrm{~A}^{\mathrm{K} 119 \mathrm{R}}$ ) and analyzed ubiquitylation of the ectop- ically expressed proteins. We found that ${ }^{\text {Flag }} \mathrm{H} 2 \mathrm{~A}$ was monoubiquitylated while ${ }^{\text {Flag }} \mathrm{H} 2 \mathrm{~A}^{\mathrm{K} 119 \mathrm{R}}$ was not (Fig. 5F). More importantly, UV exposure enhanced ubiquitylation of ${ }^{\text {Flag }} \mathrm{H} 2 \mathrm{~A}$ but did not induce ubiquitylation of ${ }^{\text {Flag }} \mathrm{H} 2 \mathrm{~A}^{\mathrm{K} 119 \mathrm{R}}$ (Fig. 5F). This indicates that the same lysine residue of $\mathrm{H} 2 \mathrm{~A}$ that is ubiquitylated under nonchallenging conditions is also targeted in DNA damage-induced monoubiquitylation.

\section{The ubiquitin ligase Ring2 is required for DNA damage-induced H2A ubiquitylation}

It has been recently reported that Ring2 is the dominant ubiquitin ligase involved in ubiquitylation of $\mathrm{H} 2 \mathrm{~A}$ on repressed DNA (de Napoles et al. 2004; Wang et al. 2004). To test whether the same ubiquitin ligase is responsible for the DNA damage-induced ubiquitylation, we generated a vector that encoded Ring2-specific RNA interference (RNAi), based on a previously published Ring2-specific RNAi construct (Wang et al. 2004), and an mRFP marker to identify cells that express the RNAi vector.
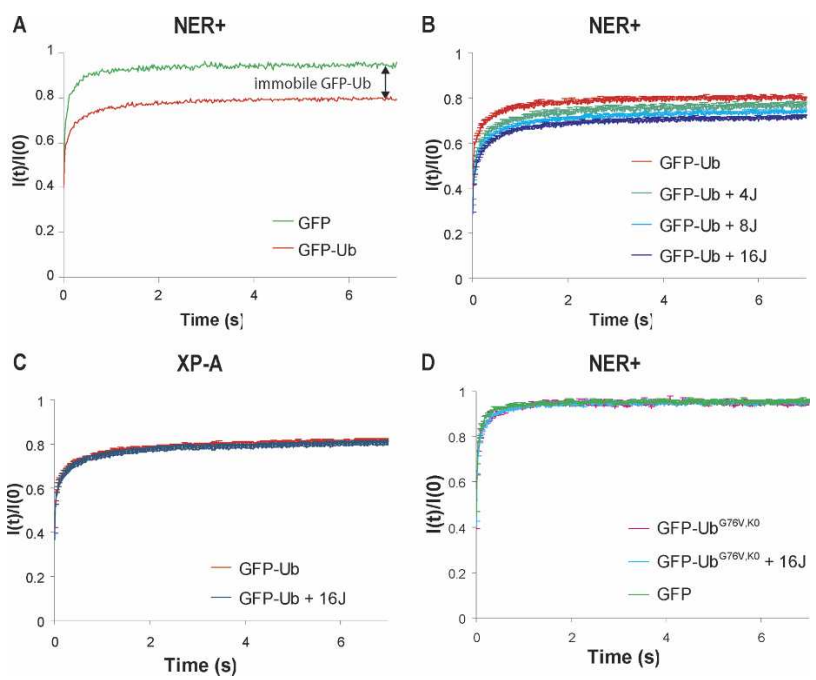

Figure 4. Global UV irradiation causes NER-dependent immobilization of nuclear ubiquitin. (A) FRAP analysis of GFP and GFP-Ub in nuclei of wild-type C5RO-hTERT cells. The immobile pool of GFP-Ub is indicated. (B) FRAP analysis of GFP-Ub in nuclei of wild-type C5RO-hTERT cells. Cells were left untreated or exposed to 4,8 , and $16 \mathrm{~J} / \mathrm{m}^{2} \mathrm{UV}$ light. Approximately $21 \%$ of the total nuclear ubiquitin pool was immobilized in wild-type C5RO-hTERT human fibroblasts. UV increased the immobile fraction in a dose-dependent manner to $24 \%, 27 \%$, and $30 \%$ immobilization after 4,8 , and $16 \mathrm{~J} / \mathrm{m}^{2}$, respectively $(n>24)$. Note that the FRAP curve of untreated GFP-Ub cells is the same data set as seen in $A$. (C) FRAP analysis of nuclear GFP-Ub in the nuclei of NER-deficient XP-A cells (XP12RO-Sv). Note that the UV damage-induced immobilization was not observed in XP-A cells (mean of 25 cells). (D) FRAP analysis of GFP-Ub $\mathrm{K}^{\mathrm{K} 0, \mathrm{G} 76 \mathrm{~V}}$ and GFP in the nuclei of wild-type C5ROhTERT cells. The dynamics of the mutant GFP-Ub ${ }^{\mathrm{K} 0, \mathrm{G} 76 \mathrm{~V}}$ and GFP (mean of 16 cells) in C5RO-hTERT cells were similar. No difference was detected in mobility before and after UV exposure (mean of 16 cells). 
Bergink et al.

Figure 5. UV irradiation causes NER-dependent ubiquitylation of histone H2A. (A) Core histones were purified before and after UV $\left(16 \mathrm{~J} / \mathrm{m}^{2}\right)$ exposure from NERproficient (MRC5-Sv) and probed with an antibody directed against ubiquitin. An increase in a $23-\mathrm{kDa}$ protein reacting with antibodies against ubiquitin was observed. $(B)$ The samples shown in $A$ probed with an antibody specific for uH2A. Blots in the bottom panels of $A$ and $B$ were stripped and reprobed with H2B-specific antibody. (C) Peptides identified by liquid chromatography tandem mass spectroscopy from the top band shown in $A$. MRC5-Sv (NER-proficient) $(D)$ and XP12RO-Sv (XP-A, NER-deficient) (E) were locally UVirradiated and stained with uH2A- and XPC-specific antibodies. uH2A accumulated in NER-proficient cells (top panels) at the damaged spot but not in XP-A cells (bottom panels). (F) Core histones were purified before and after UV $\left(16 \mathrm{~J} / \mathrm{m}^{2}\right)$ treatment from cells ectopically expressing ${ }^{\text {Flag }} \mathrm{H} 2 \mathrm{~A}$ or ${ }^{\mathrm{Flag}} \mathrm{H} 2 \mathrm{~A}^{\mathrm{K} 119 \mathrm{R}}$. Western blots probed with anti-Flag. An increase in the band corresponding to ubiquitylated ${ }^{\text {Flag }} \mathrm{H} 2 \mathrm{~A}$ was detected after UV treatment, while no ubiquitylated ${ }^{\text {Flag }} \mathrm{H} 2 \mathrm{~A}^{\mathrm{K} 119 \mathrm{R}}$ before or after UV treatment was detected.

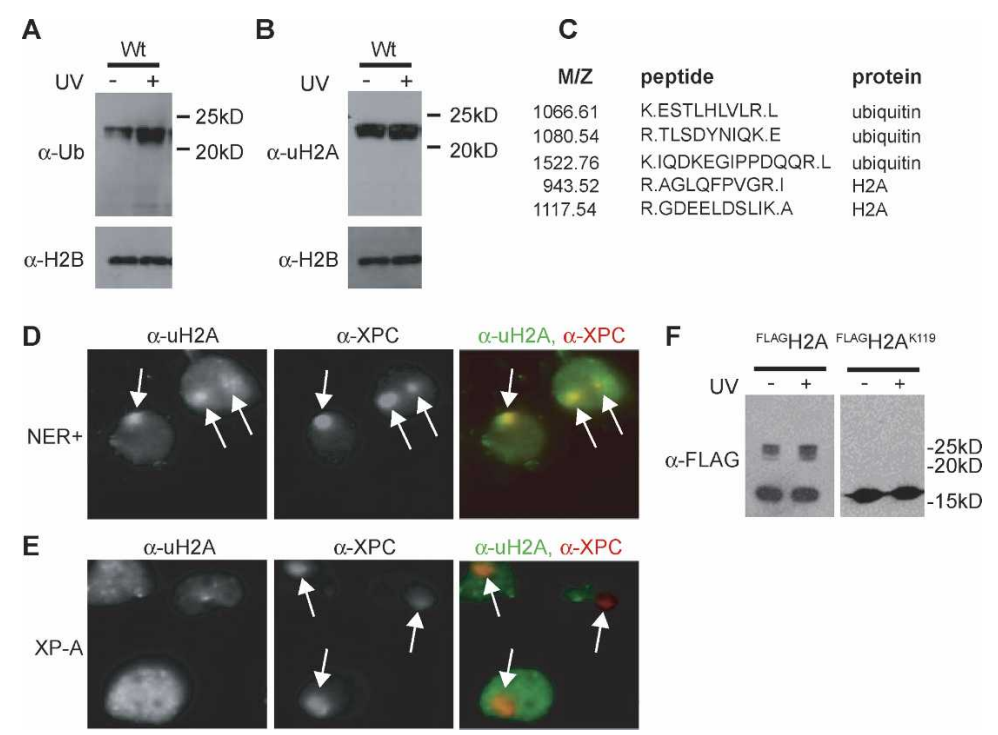

Immunostainings confirmed that this RNAi construct successfully knocked down expression of Ring2 in Mel JuSo cells (data not shown). GFP-Ub-expressing cells transfected with the RNAi construct displayed reduced levels of nuclear GFP-Ub in line with a decrease in ubiquitylated histones (Fig. 6). Importantly, the DNA damage-induced ubiquitylation was strongly reduced in the cells expressing Ring2 RNAi as compared with untransfected cells (Fig. 6). Similar results were obtained in cells with locally UV-inflicted damage (data not shown). Thus, monoubiquitylation of $\mathrm{H} 2 \mathrm{~A}$ in response to DNA damage is dependent on the H2A ubiquitin ligase Ring2.

\section{H2A ubiquitylation does not require variant histone H2AX but is dependent on ATR}

In addition to double-strand breaks also the process of NER can trigger phosphorylation of variant histone H2AX, currently the only known NER-induced histone modification (O'Driscoll et al. 2003). Immunostaining revealed that $\mathrm{uH} 2 \mathrm{~A}$ and $\gamma \mathrm{H} 2 \mathrm{AX}$ often colocalized on the local UV damage (Fig. 7A). Since it is not uncommon that different types of histone modifications are mechanistically linked (Jenuwein and Allis 2001; Zhang 2003), we tested whether ubiquitylation of $\mathrm{H} 2 \mathrm{~A}$ was dependent on NER-induced phosphorylation of H2AX. To that aim, we expressed GFP-Ub in fibroblast cells derived from H2AX-deficient and wild-type mouse embryos. These cell lines were transiently transfected with the GFP-Ub expression constructs. Both cell lines accumulated GFPUb in laser-damaged areas (Fig. 7B). Since these cells completely lack $\mathrm{H} 2 \mathrm{AX}$, we can exclude the possibility that ubiquitylation of $\mathrm{H} 2 \mathrm{~A}$ depends on phosphorylation of H2AX.

DNA damage-induced signaling is an important event in the control of the cellular response to DNA damage. The kinases ATM and ATR are important players in
DDR that are activated upon (DNA) stress and phosphorylate downstream targets (Shiloh 2003). To determine whether these kinases are important for the induc-


Figure 6. The ubiquitin ligase Ring2 is required for DNA damage-induced H2A ubiquitylation. GFP-Ub expressing Mel JuSo cells were transfected with a plasmid encoding a Ring2-specific RNAi and mRFP. (Top left panel) The transfected cells could be identified by mRFP expression (grayscale). This picture shows four representative transfected cells (asterisks) and two untransfected cells. (Top right panel) Ring2 RNAi transfected cells had reduced nuclear GFP-Ub levels (LUT [Look Up Table] color). (Bottom left panel) Laser damage was induced in two untransfected cells and two RNAi transfected cells (arrows) (LUT color). (Bottom right panel) The untransfected cells showed a clear accumulation of GFP-Ub in the laser exposed region whereas the response was very low in both Ring2 RNAi transfected cells (LUT color). The LUT for the color coding is shown at the right. Note that blue refers to overexposed on the LUT scale. 
A

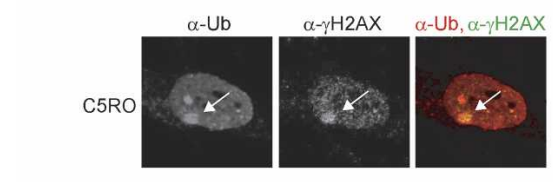

B

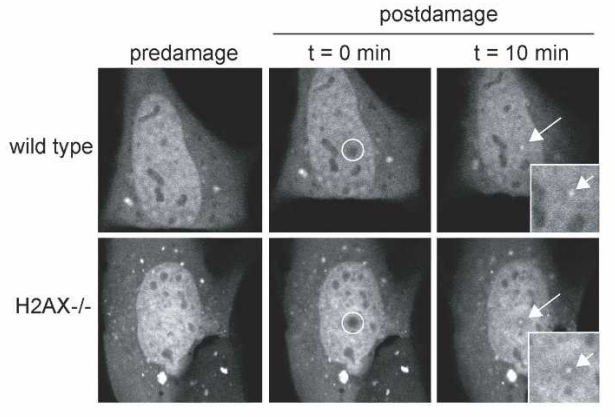

C

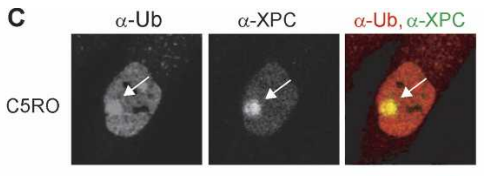

D

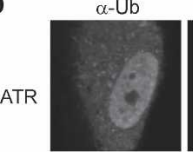

$\alpha-X P C$

\section{E}

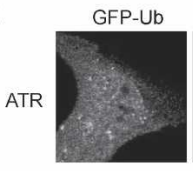

F

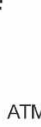

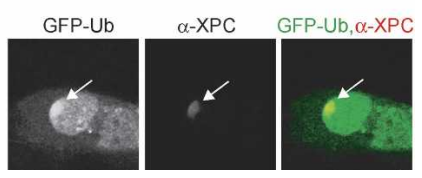

Figure 7. H2A ubiquitylation does not require variant histone H2AX but is dependent on ATR. (A) Local DNA damage was caused by UV light in CRO5 cells. Phosphorylated $\mathrm{H} 2 \mathrm{AX}$ and accumulation of ubiquitin colocalized on the lesion. $(B)$ Wild-type and $H 2 A X^{-/-}$MEF cells were transfected with GFP-Ub. After 405-nm laser damage, GFP-Ub localized at the laserirradiated areas, which are indicated by the arrows. Local DNA damage was inflicted in C5RO (NER-proficient) cells and ATR mutant cells. In C5RO cells $(C)$, but not in $A T R$ mutant cells $(D)$, endogenous ubiquitin accumulated on the lesions. ATR (E) and ATM $(F)$ mutant cell lines were transiently transfected with GFP-Ub. The lesions were identified by XPC staining. In the ATM cells, but not in the ATR cells, GFP-Ub was found enriched on the DNA damage. tion of uH2A upon UV exposure, we locally inflicted DNA damage in mutant cells of each kinase. Interestingly, whereas UV exposure triggered ubiquitin accumulation in wild-type fibroblast (Fig. 7C), accumulation of endogenous ubiquitin (Fig. 7D) or GFP-Ub (Fig. 7E) was less evident or completely absent in a patient cell line with reduced ATR expression (O'Driscoll et al. 2003). However, in cells deficient in ATM, which is primarily involved in double-strand break-induced signaling, GFPUb was still sequestered at the lesions (Fig. 7F). This indicates that DNA damage-induced signaling through ATR stimulates the induction of $\mathrm{uH} 2 \mathrm{~A}$ upon DNA damage, analogous to the situation for $\gamma \mathrm{H} 2 \mathrm{AX}$.

\section{Discussion}

In this study, we show that UV-induced DNA damage induces monoubiquitylation in close proximity to DNA lesions. We identified histone $\mathrm{H} 2 \mathrm{~A}$ as the primary target in this NER-dependent ubiquitylation response. It appears that the DNA damage-induced monoubiquitylation event is identical to the constitutive ubiquitylation of H2A involved in gene silencing, since the same target lysine residue is ubiquitylated and this ubiquitylation is dependent on Ring2, the ubiquitin ligase responsible for the bulk of H2A ubiquitylation.

To our knowledge, ubiquitylation of $\mathrm{H} 2 \mathrm{~A}$ is, next to phosphorylation of the variant histone $\mathrm{H} 2 \mathrm{AX}$, the second covalent modification of histones that is induced in an NER-dependent manner in mammalian cells. There are several striking similarities between DNA damageinduced $\gamma \mathrm{H} 2 \mathrm{AX}$ and $\mathrm{uH} 2 \mathrm{~A}$. First, both histone modifications are in UV-exposed, non-S-phase cells strictly dependent on functional NER (O'Driscoll et al. 2003). Second, the DNA damage signaling kinase ATR is required for both histone modifications. Third, similar to $\gamma \mathrm{H} 2 \mathrm{AX}$, $\mathrm{H} 2 \mathrm{~A}$ ubiquitylation spreads over a confined distance (see below), which is in the same order of magnitude as $\gamma \mathrm{H} 2 \mathrm{AX}$ (Shroff et al. 2004).
One of the most striking features of this UV-induced ubiquitylation is the extent of the modification. It is well established that ubiquitylation of several repair proteins is triggered by UV exposure; none of these ubiquitylation events passes the detection limit in our live cell imaging experiments. Although XPC, DDB2, and PCNA polyand monoubiquitylation still occur upon DNA damage in NER-deficient XP-A cell lines, we were unable to detect accumulation of ubiquitin at the DNA lesions in these mutant cells. This is in itself not surprising given the vast amount of ubiquitylated proteins in the nuclear environment. The UV-induced ubiquitylation of $\mathrm{H} 2 \mathrm{~A}$, on the contrary, induces accumulation of ubiquitin that can be readily monitored in living cells by fluorescence microscopy. The observation that global UV exposure significantly immobilizes ubiquitin in the nucleus underscores the massive nature of this event. Based on the amount of immobilized ubiquitin upon global UV irradiation and the rate of NER-mediated lesion removal, we have estimated that for each repaired DNA lesion 50150 H2A molecules are monoubiquitylated (Supplemental Material). This would correspond with nucleosomes being modified over a region of $10-30 \mathrm{~kb}$ for each lesion.

Two independent lines of evidence in our study clearly support that DNA damage $\mathrm{H} 2 \mathrm{~A}$ ubiquitylation occurred downstream from the assembly of the NER machinery. First, immunostainings show that UV-induced damage causes local accumulation of uH2A in NER-proficient cells but not in any of the NER-deficient cells, including cell lines with mutations in NER factors that act late in the reaction. Second, FRAP analysis revealed that global UV damage causes NER-dependent immobilization of a substantial pool of ubiquitin in living cells, in line with ubiquitylation of an abundant immobile nuclear target (e.g., histones). Notably, this UV-induced immobilization of ubiquitin was not observed in XP-A cells. While this paper was under review, Kapetanaki et al. (2006) reported that the UV-irradiation induced a quick reduction of $\mathrm{uH} 2 \mathrm{~A}$ and that the ligase complex DDB1- 
$\mathrm{Cul}_{4} \mathrm{~A}^{\mathrm{DDB} 2}$ was required to restore levels of $\mathrm{uH} 2 \mathrm{~A}$ at lesions, which is believed to be important for lesion recognition. Our data do not support a role for the DDB1$\mathrm{Cul}_{4} \mathrm{~A}^{\mathrm{DDB} 2}$ ubiquitin ligase in the UV-induced histone $\mathrm{H} 2 \mathrm{~A}$ ubiquitylation described herein. We observed that UV-induced H2A ubiquitylation is absent in XP-A, XP-F, $\mathrm{XP}-\mathrm{G}$, and ATR cells whereas it has been reported that ubiquitylation of other targets at the NER site of DDB1Cul4A ${ }^{\text {DDB2 }}$ still occur in these cells (Sugasawa et al. 2005). Moreover, we show that H2A ubiquitylation is induced after UV exposure and that this process is dependent on the ubiquitin ligase Ring2. It is however not excluded that different $\mathrm{H} 2 \mathrm{~A}$ ubiquitylation events are connected to the NER pathway, one mediated by DDB1Cul4A ${ }^{\mathrm{DDB} 2}$ and occurring early in NER and a Ring2-mediated induced in a late NER step of which the former has escaped notification in our system possibly due to its very transient existing.

Studies in yeast suggest a role for histone ubiquitylation in G1/S checkpoint signaling after UV-induced DNA damage (Giannattasio et al. 2005). Notably, in the yeast nucleosome the histone $\mathrm{H} 2 \mathrm{~B}$ is the primary ubiquitylation target instead of $\mathrm{H} 2 \mathrm{~A}$ as in mammalian cells. Yeast strains expressing the mutant histone $\mathrm{H} 2 \mathrm{~B}^{\mathrm{K} 123 \mathrm{R}}$ that cannot be ubiquitylated are viable and not overtly sensitive to UV light (Robzyk et al. 2000). However, UVinduced phosphorylation of Rad53 is abrogated in the $\mathrm{H} 2 \mathrm{~B}^{\mathrm{K} 123 \mathrm{R}}$ strain resulting in a failure to block DNA replication (Giannattasio et al. 2005). Thus, it is tempting to speculate that $\gamma \mathrm{H} 2 \mathrm{AX}$ and $\mathrm{uH} 2 \mathrm{~A}$ are part of the same post-repair epigenetic histone code involved in DNA damage signaling. Importantly, we found that ubiquitylation of $\mathrm{H} 2 \mathrm{~A}$ does not require the presence of $\mathrm{H} 2 \mathrm{AX}$, suggesting that these modifications are independently regulated. Given the similarities between ubiquitylation of $\mathrm{H} 2 \mathrm{~A}$ and phosphorylation of $\mathrm{H} 2 \mathrm{AX}$ and the pivotal role of $\gamma \mathrm{H} 2 \mathrm{AX}$ in double-strand break repair (Thiriet and Hayes 2005), it will be interesting to study whether ubiquitylation is restricted to NER or also plays a more general role in different forms of DNA repair.

The novel link between histone ubiquitylation and UV-induced DNA damage revealed in this study highlights the importance of nucleosome remodeling and modifications in NER. A better understanding of the significance of post-translational histone modifications in NER may shed some light on the enigmatic link between the status of histones, chromatin remodeling, and DNA repair.

\section{Materials and methods}

\section{Cell culture}

We generated stable cell lines by transfecting C5RO-hTERT (human NER-proficient fibroblast) and the SV40 immortalized human fibroblasts XP20MA-Sv (XP-C), XP12RO-Sv (XP-A), and Mel JuSo with the GFP-Ub and GFP-Ub ${ }^{\mathrm{KO}, \mathrm{G} 76 \mathrm{~V}}$ constructs. Stably expressing cells were isolated after subsequent selection of neomycin resistance and selection of GFP expression by flow cytometry-activated cell sorting. GFP-Ub and GFP-Ub ${ }^{\mathrm{KO}}$ were transiently expressed in HeLa, MRC5-Sv (NER-proficient human fibroblast), XP21RO-Sv (XP-C), XP3BR-Sv (XP-G), and
XP2YO-Sv (XP-F) cells. Cells were cultured in a mixture of Ham's F10 and Dulbecco's modified Eagle medium (GIBCOBRL) containing $10 \%$ fetal calf serum (FCS) supplemented with antibiotics at $37^{\circ} \mathrm{C}$ and $5 \% \mathrm{CO}_{2}$. Primary human fibroblasts, C5RO, C7RO (both wild-type human fibroblasts), XP25RO (XPA), XP1RO (XP-V), AT2RO (ATM), and GM18366 (fibroblasts with low level of ATR protein, obtained from Coriell Institute), used for immunofluorescence, were cultured on coverslips in Ham's F10 medium containing 15\% FCS and supplemented with antibiotics. Murine $\mathrm{H}_{2} \mathrm{AX^{-/- }}$ and wild-type embryonic fibroblasts cell lines were generated from crosses of heterozygotes $\mathrm{H}_{2} \mathrm{AX}^{+/-}$(Celeste et al. 2002) (gift from Dr. André Nussenzweig, National Cancer Institute, Bethesda, MD) (approved by ethical committee in Stockholm; permission no. N118/05). Treatment with UV light was performed with 254-nm (UV-C) light using a germicidal lamp at the indicated doses. Prior to local and overall UV irradiation, cells were rinsed with phosphate-buffered saline. For local UV irradiation, cells were grown on coverslips and were covered with 5 - or 8 - $\mu \mathrm{m}$ pore-containing membranes (Millipore) and irradiated with $65 \mathrm{~J} / \mathrm{m}^{2}$.

\section{Constructs}

The previously described RNAi sequence for Ring2 (Wang et al. 2004) was inserted in the BglII and HindIII site of pSUPERmRFP. This vector is a variant of the RNAi-expression vector pSUPER (Brummelkamp et al. 2002). The sequence of the expressed RNAi is 5'-TTCCAAAAAGGCTAGAGCTTGATAA TAATCTCTTGAATTATTATCAAGCTCTAGCCGGG-3' (Ring2 target sequence underlined). Wild-type ubiquitin (Ub), ubiquitin in which all lysine residues had been substituted by arginines $\left(\mathrm{Ub}^{\mathrm{KO}}\right)$, and ubiquitin in which all lysine residues and the terminal glycine residue had been substituted by arginines and a valine, respectively $\left(\mathrm{Ub}^{\mathrm{K} 0, \mathrm{G} 76 \mathrm{~V}}\right)$, were cloned in the vector EGFP-C1 (Clontech) using the restriction sites Asp718 and HindIII. The GFP-PCNA construct is a gift from Dr. Jeroen Essers (Erasmus Medical Center, Rotterdam, The Netherlands). ${ }^{\text {Flag }}$ H2A was obtained by subcloning GFP-H2A (a gift from Dr. Pierre-Yeves Perche, INSERM U309, Institut Albert Bonniot, La Tronche cedex, France) in pcDNA3.1Flag. The codon encoding Lys 119 in Flag H2A was substituted for an arginine codon with QuikChange XL site-directed mutagenesis (Stratagene) using the following primers: K119R Fw: 5'-CGTGCTACTGCCCA AGAgGACCGAGAGCCACCACAAGG-3'; Rv: 5' -CCTTGTG GTGGCTCTCGGTCCTCTTGGGCAGTAGCACG-3' .

\section{Immunofluorescence microscopy}

Cells were fixed with $2 \%$ paraformaldehyde in the presence of $0.2 \%$ Triton X-100. For uH2A antibody staining, cells were incubated with $0.5 \%$ Triton X-100 prior to fixation (de Napoles et al. 2004). Samples were processed as described previously (Rademakers et al. 2003). Immunofluoresecent images were obtained by the Aristoplan Flu 134,795 (Leitz). The following antibodies were used: anti-hXPC (rabbit polyclonal anti-human XPC), antiCPD (gift from Dr. O. Nikaido, Kanazawa University, Kanazawa, Japan), anti-uH2A (Upstate Biotechnology 05-678), $20 \mathrm{~S}$ sampler pack (Biomol), anti-ubiquitin (FK2; Affiniti), anti$\gamma \mathrm{H} 2 \mathrm{AX}$ (Upstate Biotechnology 07-164), anti-Ring2 (gift from Dr. M. van Lohuizen, The Netherlands Cancer Institute, Amsterdam, The Netherlands), and corresponding secondary antibodies (Molecular Probes and Jackson Laboratory).

\section{Live cell confocal laser scanning microscopy}

Confocal laser scanning microscopy images were obtained on a Zeiss LSM 510 META equipped with cell culture microscopy 
stage. GFP fluorescence imaging was recorded after excitation with a 488-nm argon laser and a 515-540-nm band-pass filter. Fluorescent redistribution after photobleaching was performed as described previously (Houtsmuller and Vermeulen 2001). Fluorescence within a $0.8-\mu \mathrm{m}$ strip spanning the nucleus was monitored every $20.5 \mathrm{msec}$ using a Zeiss LSM 510 META and a 488-nm argon laser excitation at $0.5 \%$ transmission; after 200 measurements this strip was photobleached using one iteration at $100 \%$ transmission and the influx fluorescence was recorded for another 1000 measurements. The obtained fluorescence recovery curves were normalized to the prebleach fluorescence set at 1 . For live cell imaging of laser-induced DNA damage, cells were irradiated in a spot using $100 \% 405 \mathrm{~nm}$ for 2000 iterations. Directly after irradiation cells were monitored every minute for $10 \mathrm{~min}$. Fluorescent intensities were measured using ImageJ software. Accumulation was calculated by averaging the fluorescent ratio between exposed and nonexposed areas in the nucleus.

\section{Histone purification}

Cells were harvested $30 \mathrm{~min}$ post-irradiation $\left(16 \mathrm{~J} / \mathrm{m}^{2}\right.$ of UV) or mock treatment by scraping and subsequent centrifugation in ice-cold phosphate-buffered saline. Histonel and high-mobility group proteins were isolated using $5 \%$ perchloric acid extraction. Extraction was repeated three times; the supernatants were separated from the pellet by centrifugation. From the remaining pellet core histones were dissolved in $0.4 \mathrm{M} \mathrm{HCl}$; extraction was repeated three times. Pellet and supernatant were separated using centrifugation. Pooled supernatants were precipitated using $25 \%$ trichloroacetic acid. After precipitation pellets were washed with acetone and dried using a speedvac (Giancotti et al. 1985). All steps were performed at $4^{\circ} \mathrm{C}$. All buffers contained protease inhibitor cocktail (chymostatin, leupin, antipain, and pepstatin A) and $10 \mathrm{mM}$ iodoacetamide to inhibit deubiquitylation activity. Samples were run on $15 \%$ polyacrylamide gels and transferred to $0.1-\mu \mathrm{m}$ pore nitrocellulose membranes. Membranes were incubated with anti-H2A (Upstate Biotechnology 07-146), anti-H2B (Upstate Biotechnology 07-266), anti-ubiquitin (FK2), or antiFlag (Sigma) followed by corresponding secondary antibody incubation (Amersham NA934, DAKO P0161, or Biosource AMI4604) and detected using enhanced chemiluminescence (Amersham).

\section{Acknowledgments}

We thank Pier Paolo Di Fiore for the stay of S.B. in his laboratory; André Nussenzweig, Jeroen Essers, and Pierre-Yeves Perche for mice and reagents; Arjan Theil, Jeroen A.A. Demmers, and K. Bestaroszti for technical assistance; and Lisette Verhoef and Victoria Menéndez-Benito for critical reading of the manuscript. This work was supported by grants of the Netherlands Organisation for Scientific Research (NWO: 014-90-001 [to S.B.], 805-33-441-P [to D.H.], 912-03-12/917-46-364 [to W.V.]; EC: LSHG-CT-2005-512113 [to H.dW.]), the Swedish Research Council (to N.P.D.), Swedish Cancer Society (to N.P.D.), the Nordic Center of Excellence Neurodegeneration (to N.P.D.), and the Karolinska Institute (to N.P.D.). The Netherlands Organisation for Scientific Research (NWO) supported a sabbatical stay of N.P.D. in the laboratory of J.N. during which the initial observations in this study were made. N.P.D. is supported by the Swedish Research Council. We declare that there is no conflict of interest.

\section{References}

Araki, M., Masutani, C., Takemura, M., Uchida, A., Sugasawa, K., Kondoh, J., Ohkuma, Y., and Hanaoka, F. 2001. Centrosome protein centrin $2 /$ caltractin 1 is part of the xeroderma pigmentosum group $\mathrm{C}$ complex that initiates global genome nucleotide excision repair. J. Biol. Chem. 276: 18665-18672.

Brummelkamp, T.R., Bernards, R., and Agami, R. 2002. A system for stable expression of short interfering RNAs in mammalian cells. Science 296: 550-553.

Celeste, A., Petersen, S., Romanienko, P.J., Fernandez-Capetillo, O., Chen, H.T., Sedelnikova, O.A., Reina-San-Martin, B., Coppola, V., Meffre, E., Difilippantonio, M.J., et al. 2002. Genomic instability in mice lacking histone H2AX. Science 296: 922-927.

Citterio, E., Vermeulen, W., and Hoeijmakers, J.H. 2000. Transcriptional healing. Cell 101: 447-450.

Cleaver, J.E. 2005. Cancer in xeroderma pigmentosum and related disorders of DNA repair. Nat. Rev. Cancer 5: 564-573.

Dantuma, N.P., Groothuis, T.A. Salomons, F.A., and Neefjes, J. 2006. A dynamic ubiquitin equilibrium couples proteasomal activity to chromatin remodeling. J. Cell. Biol. 173: 19-26.

de Laat, W.L., Jaspers, N.G., and Hoeijmakers, J.H. 1999. Molecular mechanism of nucleotide excision repair. Genes \& Dev. 13: 768-785.

de Napoles, M., Mermoud, J.E., Wakao, R., Tang, Y.A., Endoh, M., Appanah, R., Nesterova, T.B., Silva, J., Otte, A.P., Vidal, M., et al. 2004. Polycomb group proteins Ring1A/B link ubiquitylation of histone $\mathrm{H} 2 \mathrm{~A}$ to heritable gene silencing and $\mathrm{X}$ inactivation. Dev. Cell 7: 663-676.

Essers, J., Theil, A.F., Baldeyron, C., van Cappellen, W.A., Houtsmuller, A.B., Kanaar, R., and Vermeulen, W. 2005. Nuclear dynamics of PCNA in DNA replication and repair. Mol. Cell. Biol. 25: 9350-9359.

Giancotti, V., Berlingieri, M.T., DiFiore, P.P., Fusco, A., Vecchio, G., and Crane-Robinson, C. 1985. Changes in nuclear proteins on transformation of rat epithelial thyroid cells by a murine sarcoma retrovirus. Cancer Res. 45: 6051-6057.

Giannattasio, M., Lazzaro, F., Plevani, P., and Muzi-Falconi, M. 2005. The DNA damage checkpoint response requires histone $\mathrm{H} 2 \mathrm{~B}$ ubiquitination by Rad6-Bre1 and $\mathrm{H} 3$ methylation by Dot1. J. Biol. Chem. 280: 9879-9886.

Glickman, M.H. and Ciechanover, A. 2002. The ubiquitin-proteasome proteolytic pathway: Destruction for the sake of construction. Physiol. Rev. 82: 373-428.

Green, C.M. and Almouzni, G. 2002. When repair meets chromatin. First in series on chromatin dynamics. EMBO Rep. 3: 28-33.

Hoege, C., Pfander, B., Moldovan, G.L., Pyrowolakis, G., and Jentsch, S. 2002. RAD6-dependent DNA repair is linked to modification of PCNA by ubiquitin and SUMO. Nature 419: 135-141.

Hoeijmakers, J.H. 2001. Genome maintenance mechanisms for preventing cancer. Nature 411: 366-374.

Houtsmuller, A.B. and Vermeulen, W. 2001. Macromolecular dynamics in living cell nuclei revealed by fluorescence redistribution after photobleaching. Histochem. Cell Biol. 115: $13-21$.

Jenuwein, T. and Allis, C.D. 2001. Translating the histone code. Science 293: 1074-1080.

Kannouche, P.L., Wing, J., and Lehmann, A.R. 2004. Interaction of human DNA polymerase eta with monoubiquitinated PCNA: A possible mechanism for the polymerase switch in response to DNA damage. Mol. Cell 14: 491-500.

Kapetanaki, M.G., Guerrero-Santoro, J., Bisi, D.C., Hsieh, C.L., Rapic-Otrin, V., and Levine, A.S. 2006. The DDB1- 
Bergink et al.

CUL4ADDB2 ubiquitin ligase is deficient in xeroderma pigmentosum group E and targets histone $\mathrm{H} 2 \mathrm{~A}$ at UV-damaged DNA sites. Proc. Natl. Acad. Sci. 103: 2588-2593.

Katsumi, S., Kobayashi, N., Imoto, K., Nakagawa, A., Yamashina, Y., Muramatsu, T., Shirai, T., Miyagawa, S., Sugiura, S., Hanaoka, F., et al. 2001. In situ visualization of ultraviolet-light-induced DNA damage repair in locally irradiated human fibroblasts. J. Invest. Dermatol. 117: 1156-1161.

Leonhardt, H., Rahn, H.P., Weinzierl, P., Sporbert, A., Cremer, T., Zink, D., and Cardoso, M.C. 2000. Dynamics of DNA replication factories in living cells. J. Cell Biol. 149: 271-280.

Levinger, L. and Varshavsky, A. 1982. Selective arrangement of ubiquitinated and D1 protein-containing nucleosomes within the Drosophila genome. Cell 28: 375-385.

Masutani, C., Kusumoto, R., Yamada, A., Dohmae, N., Yokoi, M., Yuasa, M., Araki, M., Iwai, S., Takio, K., and Hanaoka, F. 1999. The XPV (xeroderma pigmentosum variant) gene encodes human DNA polymerase eta. Nature 399: 700-704.

Nickel, B.E. and Davie, J.R. 1989. Structure of polyubiquitinated histone H2A. Biochemistry 28: 964-968.

O’Driscoll, M., Ruiz-Perez, V.L., Woods, C.G., Jeggo, P.A., and Goodship, J.A. 2003. A splicing mutation affecting expression of ataxia-telangiectasia and Rad3-related protein (ATR) results in Seckel syndrome. Nat. Genet. 33: 497-501.

Peterson, C.L. and Cote, J. 2004. Cellular machineries for chromosomal DNA repair. Genes \& Dev. 18: 602-616.

Qian, S.B., Ott, D.E., Schubert, U., Bennink, J.R., and Yewdell, J.W. 2002. Fusion proteins with $\mathrm{COOH}$-terminal ubiquitin are stable and maintain dual functionality in vivo. J. Biol. Chem. 277: 38818-38826.

Rademakers, S., Volker, M., Hoogstraten, D., Nigg, A.L., Mone, M.J., Van Zeeland, A.A., Hoeijmakers, J.H., Houtsmuller, A.B., and Vermeulen, W. 2003. Xeroderma pigmentosum group A protein loads as a separate factor onto DNA lesions. Mol. Cell. Biol. 23: 5755-5767.

Robzyk, K., Recht, J., and Osley, M.A. 2000. Rad6-dependent ubiquitination of histone H2B in yeast. Science 287: 501504.

Rogakou, E.P., Pilch, D.R., Orr, A.H., Ivanova, V.S., and Bonner, W.M. 1998. DNA double-stranded breaks induce histone H2AX phosphorylation on serine 139. J. Biol. Chem. 273: 5858-5868.

Shiloh, Y. 2003. ATM and related protein kinases: Safeguarding genome integrity. Nat. Rev. Cancer 3: 155-168.

Shroff, R., Arbel-Eden, A., Pilch, D., Ira, G., Bonner, W.M., Petrini, J.H., Haber, J.E., and Lichten, M. 2004. Distribution and dynamics of chromatin modification induced by a defined DNA double-strand break. Curr. Biol. 14: 1703-1711.

Smerdon, M.J. 1991. DNA repair and the role of chromatin structure. Curr. Opin. Cell Biol. 3: 422-428.

Sugasawa, K., Ng, J.M., Masutani, C., Iwai, S., van der Spek, P.J., Eker, A.P., Hanaoka, F., Bootsma, D., and Hoeijmakers, J.H. 1998. Xeroderma pigmentosum group C protein complex is the initiator of global genome nucleotide excision repair. Mol. Cell 2: 223-232.

Sugasawa, K., Okuda, Y., Saijo, M., Nishi, R., Matsuda, N., Chu, G., Mori, T., Iwai, S., Tanaka, K., and Hanaoka, F. 2005. UV-induced ubiquitylation of XPC protein mediated by UVDDB-ubiquitin ligase complex. Cell 121: 387-400.

Thiriet, C. and Hayes, J.J. 2005. Chromatin in need of a fix: Phosphorylation of H2AX connects chromatin to DNA repair. Mol. Cell 18: 617-622.

Thrower, J.S., Hoffman, L., Rechsteiner, M., and Pickart, C.M. 2000. Recognition of the polyubiquitin proteolytic signal. EMBO J. 19: 94-102.

Vidanes, G.M., Bonilla, C.Y., and Toczyski, D.P. 2005. Compli- cated tails: Histone modifications and the DNA damage response. Cell 121: 973-976.

Wang, H., Wang, L., Erdjument-Bromage, H., Vidal, M., Tempst, P., Jones, R.S., and Zhang, Y. 2004. Role of histone H2A ubiquitination in Polycomb silencing. Nature 431: 873-878.

Wang, Q.E., Wani, M.A., Chen, J., Zhu, Q., Wani, G., El-Mahdy, M.A., and Wani, A.A. 2005. Cellular ubiquitination and proteasomal functions positively modulate mammalian nucleotide excision repair. Mol. Carcinog. 42: 53-64.

Zhang, Y. 2003. Transcriptional regulation by histone ubiquitination and deubiquitination. Genes \& Dev. 17: 2733-2740. 


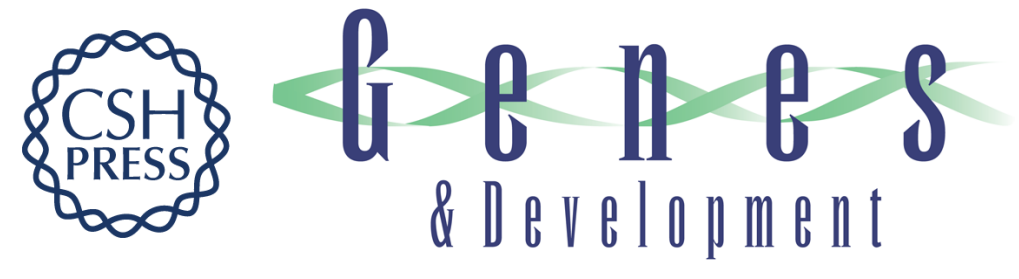

\section{DNA damage triggers nucleotide excision repair-dependent monoubiquitylation of histone $\mathrm{H} 2 \mathrm{~A}$}

Steven Bergink, Florian A. Salomons, Deborah Hoogstraten, et al.

Genes Dev. 2006, 20:

Access the most recent version at doi:10.1101/gad.373706

Supplemental http://genesdev.cshlp.org/content/suppl/2006/04/27/20.10.1343.DC1
Material

References This article cites 41 articles, 19 of which can be accessed free at:

http://genesdev.cshlp.org/content/20/10/1343.full.html\#ref-list-1

License

Email Alerting

Receive free email alerts when new articles cite this article - sign up in the box at the top

Service

right corner of the article or click here.

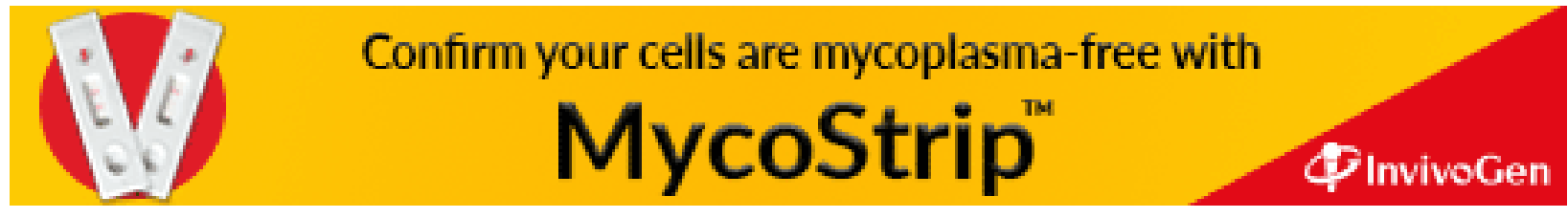

